\title{
THE GRUNSKY COEFFICIENTS OF A SCHLICHT FUNCTION
}

\author{
J. A. HUMMEL ${ }^{1}$
}

A necessary and sufficient condition for a meromorphic function to be schlicht in a given domain was obtained by Grunsky in 1939 [2]. Somewhat later, Schiffer showed how the coefficients in this condition could be obtained from the coefficients of $\log [f(z)-f(\zeta)] /[z-\zeta]$, [3]. In the particular case of functions which are schlicht in $|z|<1$, the Grunsky results, combined with those of Schiffer, can be summarized as:

THEOREM 1. A necessary and sufficient condition that $f(z)=z$ $+a_{2} z^{2}+\cdots$, which is regular in $|z|<1$, be schlicht in $|z|<1$ is that

$$
\left|\sum_{n, m=1}^{\infty} x_{n} x_{m} C_{n m}\right| \leqq \sum_{n=1}^{\infty}\left|x_{n}\right|^{2} / n
$$

for every sequence $\left\{x_{n}\right\}$ for which the right-hand side converges, where the coefficients $C_{n m}$ are determined by

$$
\log \frac{f(z)-f(\zeta)}{z-\zeta}=\sum_{n, m=0}^{\infty} C_{n m} z^{n} \zeta^{m}
$$

Recently, Charzynski and Schiffer gave a new proof that $\left|a_{4}\right| \leqq 4$ making use of essentially the Grunsky inequalities [1]. This proof, which is completely elementary except for the use of these inequalities, has caused renewed interest in the Grunsky inequalities, but a nontrivial difficulty in such a study is the actual determination of the coefficients $C_{n m}$. The main purpose of this note is to derive a formula for these coefficients. However, before proceeding on this there are a few observations of a more or less transparent nature which are worth making.

First, notice that $f(z)$ defines $C_{n m}$ for all non-negative pairs $(n, m)$. However, the Grunsky inequalities of Theorem 1 only make use of the $C_{n m}$ for which $n, m \geqq 1$. Since $g(z)=f(z) /\left[1-\left(f(z) / w_{0}\right)\right]$ has the same set of $C_{n m}$ as $f(z)$ for $n, m \geqq 1$ for any value of $w_{0} \neq 0$, the fact that $f(z)$ is regular in $|z|<1$ is a hypothesis in Theorem 1 and cannot be proved from the existence of the inequalities.

Presented to the Society, January 27, 1963; received by the editors September 8, 1962.

1 This research was supported by the National Science Foundation, under research grant NSF-G 11592 with the University of Maryland. 
Likewise the function $F(z)=1 / f(1 / z)=z+a_{0}^{\prime}+a_{1}^{\prime} / z+\cdots$, which is schlicht in $|z|>1$ if and only if $f(z)$ is schlicht in $|z|<1$, has the same set of $C_{n m}(n, m \geqq 1)$ as $f(z)$. This is the type of function actually considered by Grunsky and Schiffer, and the above observation is needed to convert their results to the form of Theorem 1.

The result of Charzynski and Schiffer is obtained by use of Theorem 1 as applied to the function $\left[f\left(z^{2}\right)\right]^{1 / 2}$. It is a well-known fact that if $f(z)=z+a_{2} z^{2}+\cdots$ is regular and schlicht in $|z|<1$, then so is $\left[f\left(z^{p}\right)\right]^{1 / p}$ for each positive integral $p$.

DEFINITION 1. Given $f(z)=z+a_{2} z^{2}+\cdots$, regular and schlicht in $|z|<1$, the Grunsky coefficients of $f(z)$ are the coefficients $C_{n m}^{(p)}, n, m, p \geqq 1$ defined by

$$
\log \frac{\left[f\left(z^{p}\right)\right]^{1 / p}-\left[f\left(\zeta^{p}\right)\right]^{1 / p}}{z-\zeta}=\sum_{n, m=0}^{\infty} C_{n m}^{(p)} z^{n} \zeta^{m} .
$$

With this definition, the inequalities of Theorem 1 can be extended to

THEOREM 2. If $f(z)=z+a_{2} z^{2}+\cdots$ is regular and schlicht in $|z|<1$, then

$$
\left|\sum_{n, m=1}^{\infty} x_{n} x_{m} C_{n m}^{(p)}\right| \leqq \sum_{n=1}^{\infty} \frac{\left|x_{n}\right|^{2}}{n}
$$

for every positive integer $p$ and every sequence $\left\{x_{n}\right\}$ such that the righthand side converges.

From this it is only a short step to the following somewhat simpler set of inequalities:

THEOREM 3. If $f(z)=z+a_{2} z^{2}+\cdots$ is regular and schlicht in $|z|<1$, then for every $k$ and every $p$

$$
\left|\sum_{n=1}^{\infty} x_{n} C_{n k}^{(p)}\right| \leqq\left[\frac{1}{k} \sum_{n=1}^{\infty} \frac{\left|x_{n}\right|^{2}}{n}\right]^{1 / 2}
$$

and

$$
\sum_{n=1}^{\infty} n\left|C_{n k}^{(p)}\right|^{2} \leqq \frac{1}{k}
$$

REMARK. Inequality (6) appears to be essentially equivalent to formulas obtained by Wolibner [5]. In this paper, Wolibner generalizes results of Biernacki and Golusin to obtain this result. have

Proof. From (3) it is obvious that $C_{n m}^{(p)}=C_{m n}^{(p)}$, hence from (4) we 


$$
\begin{aligned}
& \left|\sum_{n, m=1}^{\infty} x_{n} y_{m} C_{n m}^{(p)}\right| \\
& =\frac{1}{4}\left|\sum_{n, m=1}^{\infty}\left[\left(x_{n}+y_{n}\right)\left(x_{m}+y_{m}\right)-\left(x_{n}-y_{n}\right)\left(x_{m}-y_{m}\right)\right] C_{n m}^{(p)}\right| \\
& \leqq \frac{1}{4}\left|\sum_{n, m=1}^{\infty}\left(x_{n}+y_{n}\right)\left(x_{m}+y_{m}\right) C_{n m}^{(p)}\right|+\frac{1}{4}\left|\sum_{n, m=1}^{\infty}\left(x_{n}-y_{n}\right)\left(x_{m}-y_{m}\right) C_{n m}^{(p)}\right| \\
& \leqq \frac{1}{4} \sum_{n=1}^{\infty} \frac{\left|x_{n}+y_{n}\right|^{2}}{n}+\frac{1}{4} \sum_{n=1}^{\infty} \frac{\left|x_{n}-y_{n}\right|^{2}}{n} \\
& =\sum_{n=1}^{\infty} \frac{\left|x_{n}\right|^{2}+\left|y_{n}\right|^{2}}{2 n} .
\end{aligned}
$$

In this relation, set $y_{m}=0$ for $m \neq k$ and let $x_{n}=\xi_{n} / y_{k}$. Then

$$
\left|\sum_{n=1}^{\infty} \xi_{n} C_{n k}^{(p)}\right| \leqq \frac{1}{2} \frac{1}{\left|y_{n}\right|^{2}} \sum_{n=1}^{\infty} \frac{\left|\xi_{n}\right|^{2}}{n}+\frac{1}{2} \frac{\left|y_{k}\right|^{2}}{k} .
$$

The right-hand side of this inequality is minimized by setting $\left|y_{k}\right|^{2}$ $=\left[k \sum_{n=1}^{\infty}\left|\xi_{n}\right|^{2} / n\right]^{1 / 2}$, giving (5).

Inequality (6) can be obtained directly from the above formula if we set $y_{m}=0$ for $m \neq k, y_{k}=1$, and $x_{n}=n C_{n k}^{(p) *}$ where the asterisk indicates the complex conjugate of the term.

We now turn to the problem of determining the Grunsky coefficients $C_{n m}^{(p)}$. From (3)

$$
\begin{aligned}
\sum_{n, m=0}^{\infty} C_{n m}^{(p)} z^{n} \zeta^{m} & =\log \frac{\left[f\left(z^{p}\right)\right]^{1 / p}-\left[f\left(\zeta^{p}\right)\right]^{1 / p}}{z-\zeta} \\
& =\log \left[1-\frac{f^{1 / p}\left(z^{p}\right)}{f^{1 / p}\left(\zeta^{p}\right)}\right]-\log \left[1-\frac{z}{\zeta}\right]+\log \frac{f^{1 / p}\left(\zeta^{p}\right)}{\zeta} \\
& =-\sum_{\nu=1}^{\infty} \frac{1}{\nu}\left[\frac{f\left(z^{p}\right)}{f\left(\zeta^{p}\right)}\right]^{\nu / p}+\sum_{\nu=1}^{\infty} \frac{1}{\nu}\left[\frac{z}{\zeta}\right]^{\nu}+\log \frac{f^{1 / p}\left(\zeta^{p}\right)}{\zeta} .
\end{aligned}
$$

These series will converge and give the required representation if $\zeta$ is sufficiently small (but $\zeta \neq 0$ ) and then $z$ is sufficiently small with respect to $\zeta$. The final two terms in the last expression make no contribution to $C_{n m}^{(p)}$ for $n>0$ and $m>0$. Hence $C_{n m}^{(p)}$ will be the coefficient of $z^{n} \zeta^{m}$ in the first term.

Let us define $b_{v}=a_{v+1}$. Then

$$
f(z)=z+a_{2} z^{2}+\cdots=z\left(1+b_{1} z+b_{2} z^{2}+\cdots\right)
$$

and $\left[f\left(z^{p}\right)\right]^{p / p}=z^{p}\left(1+b_{1} z^{p}+b_{2} z^{2 p}+\cdots\right)^{p / p}$. This will contain a non- 
zero coefficient of $z^{n}$ if and only if $n=\nu+k p$ for some non-negative integer $k$. But in this case, the coefficient of $z^{n}$ in $\left[f\left(z^{p}\right)\right]^{p / p}$ will be the same as the coefficient of $z^{k}$ in $\left(1+b_{1} z+b_{2} z^{2}+\cdots\right)^{v / p}$. However, from the multinomial theorem, this coefficient will be

$$
b_{k}^{(\nu / p)}=\sum_{S_{k}}\left(\begin{array}{c}
\nu / p \\
s_{1}, s_{2}, \cdots, s_{k}
\end{array}\right) b_{1}^{s_{1}} b_{2}^{s_{2}} \cdots b_{k}^{s_{k}}
$$

where

$$
s_{k}=\left\{\left(s_{1}, s_{2}, \cdots, s_{k}\right) \mid s_{\nu} \geqq 0 \text { and } s_{1}+2 s_{2}+3 s_{3}+\cdots+k s_{k}=k\right\}
$$

and if $s_{1}+s_{2}+\cdots+s_{k}=t$, the multinomial symbol is defined to be

$$
\left(\begin{array}{c}
\nu / p \\
s_{1}, s_{2}, \cdots, s_{k}
\end{array}\right)=\frac{(\nu / p)(\nu / p-1) \cdots(\nu / p-t+1)}{s_{1} ! s_{2} ! \cdots s_{k} !} .
$$

In exactly the same way, we see that the coefficient of $\zeta^{m}$ in $\left[f\left(\zeta^{p}\right)\right]^{-\nu / p}$ is nonzero if and only if $m=-\nu+j p$ for some non-negative integer $j$, and then it is

$$
b_{j}^{(-\nu / p)}=\sum_{Q_{j}}\left(\begin{array}{c}
-\nu / p \\
r_{1}, \cdots, r_{j}
\end{array}\right) b_{1}^{r_{1}} b_{2}^{r_{2}} \cdots b_{j}^{r_{j}},
$$

where

$$
R_{j}=\left\{\left(r_{1}, r_{2}, \cdots, r_{j}\right) \mid r_{\nu} \geqq 0 \text { and } r_{1}+2 r_{2}+\cdots+j r_{j}=j\right\} \text {. }
$$

Therefore, the coefficient of $z^{n \zeta^{m}}$ in $(1 / \nu)\left[f\left(z^{p}\right) / f\left(\zeta^{p}\right)\right]^{p / p}$ will be $(1 / \nu) b_{k}^{(\nu / p)} b_{j}^{(-\nu / p)}$ providing $n=\nu+k p, m=-\nu+j p$ and will be zero otherwise. To find $C_{n m}^{(p)}$ we must sum these expressions on $\nu$. Notice that if $m+n$ is not divisible by $p$, there will be no $\nu$ with a nonzero coefficient of $z^{n} \zeta^{m}$. However, if $m+n=h p$, then we must have $k+j=h$, so we may set $\nu=n-k p$ and $j=h-k$. We must have $\nu \geqq 1, k \geqq 0, j \geqq 0$ so we may sum on $k$ from $k=0$ to the smaller of $h$ and $[n / p]$, which is $[n / p]$, except that if $n / p$ is an integer, the sum will extend only to $k=n / p-1$. Therefore we have,

$$
C_{n m}^{(p)}=-\sum_{k=0}^{[n / p]} \sum_{S_{k}} \sum_{R_{h-k}} \frac{1}{(n-k p)}\left(\begin{array}{c}
n / p-k \\
s_{1}, s_{2}, \cdots, s_{k}
\end{array}\right)\left(\begin{array}{c}
k-n / p \\
r_{1}, r_{2}, \cdots, r_{h-k}
\end{array}\right)
$$

if $n+m=h p$, and $C_{n m}^{(p)}=0$ otherwise.

In this expression, we will leave the upper limit $[n / p]$ with the understanding that if $k=n / p$ the term will be taken to be zero. Notice that in this case, the two multinomial symbols both have a zero. 
Now in any term in this expression $s_{1}+2 s_{2}+\cdots+k s_{k}=k$ and $r_{1}+2 r_{2}+\cdots+(h-k) r_{h-k}=h-k$. Hence $\left(s_{1}+r_{1}\right)+2\left(s_{2}+r_{2}\right)+\cdots$ $+h\left(s_{h}+r_{h}\right)=h$. Therefore if we set $q_{\nu}=s_{\nu}+r_{\nu}, \nu=1,2, \cdots, h$ (taking $s_{\nu}=0$ when $\nu>k$, and $r_{\nu}=0$ when $\nu>h-k$ ), we must have

$$
C_{n m}^{(p)}=\sum_{Q_{h}} C_{n m}^{(p)}\left(q_{1}, \cdots, q_{h}\right) b_{1}^{q_{1}} b_{2}^{\ell_{2}} \cdots b_{h}^{q_{h}}, \quad n+m=h p,
$$

where

$$
Q_{h}=\left\{\left(q_{1}, \cdots, q_{h}\right) \mid q_{v} \geqq 0 \text { and } q_{1}+2 q_{2}+\cdots+h q_{h}=h\right\},
$$

and

$$
\begin{aligned}
C_{n m}^{(p)}\left(q_{1}, \cdots, q_{h}\right) & \\
\quad & -\sum_{k=0}^{[n / p]} \sum_{S_{k}^{\prime}} \frac{1}{(n-k p)}\left(\begin{array}{c}
n / p-k \\
s_{1}, \cdots, s_{h}
\end{array}\right)\left(\begin{array}{c}
k-n / p \\
q_{1}-s_{1}, \cdots, q_{h}-s_{h}
\end{array}\right)
\end{aligned}
$$

where

$$
\delta_{k}^{\prime}=\left\{\left(s_{1}, s_{2}, \cdots, s_{h}\right) \mid 0 \leqq s_{\nu} \leqq q_{\nu}, s_{1}+2 s_{2}+\cdots+h s_{h}=k\right\} .
$$

Now if we let

$$
s_{1}+s_{2}+\cdots+s_{h}=t
$$

and

$$
q_{1}+q_{2}+\cdots+q_{h}=q,
$$

then (supposing $n / p \neq k$ )

$$
\begin{aligned}
& -\frac{1}{(n-k p)}\left(\begin{array}{c}
n / p-k \\
s_{1}, \cdots, s_{h}
\end{array}\right)\left(\begin{array}{c}
k-n / p \\
q_{1}-s_{1}, \cdots, q_{h}-s_{h}
\end{array}\right) \\
& =-\frac{\left(\frac{n}{p}-k\right)\left(\frac{n}{p}-k-1\right) \cdots\left(\frac{n}{p}-k-t+1\right)\left(k-\frac{n}{p}\right)\left(k-\frac{n}{p}-1\right) \cdots\left(k-\frac{n}{p}-q+t+1\right)}{p\left(\frac{n}{p}-k\right) s_{1} ! s_{2} ! \cdots s_{h} !\left(q_{1}-s_{1}\right) !\left(q_{2}-s_{2}\right) ! \cdots\left(q_{h}-s_{h}\right) !} \\
& =-\frac{(-1)^{\alpha-t}\left(\frac{n}{p}-k+q-t-1\right)\left(\frac{n}{p}-k+q-t-2\right) \cdots\left(\frac{n}{p}-k\right)\left(\frac{n}{p}-k-1\right) \cdots}{p s_{1} ! s_{2} ! \cdots s_{h} !\left(q_{1}-s_{1}\right) !\left(q_{2}-s_{2}\right) ! \cdots\left(q_{h}-s_{h}\right) !}\left(\frac{n}{p}-k-t+1\right) \\
& =(-1) r-t+1 \\
& \frac{1}{p\left(\frac{n}{p}-k+q-t\right)}\left(\begin{array}{c}
n / p-k+q-t \\
s_{1}, \cdots, s_{h}, q_{1}-s_{1}, \cdots, q_{h}-s_{h}
\end{array}\right) .
\end{aligned}
$$

We have therefore shown 
TheOREM 4. $C_{n m}^{(p)}=0$ if $n+m$ is not divisible by $p$. If $n+m=h p$ then, setting $f(z)=z\left(1+b_{1} z+b_{2} z^{2}+\cdots\right)$

where

$$
C_{n m}^{(p)}=\sum_{Q_{h}} C_{n m}^{(p)}\left(q_{1}, \cdots, q_{h}\right) b_{1}^{q_{1}} b_{2}^{q_{2}} \cdots b_{h}^{q_{h}},
$$

$$
Q_{h}=\left\{\left(q_{1}, \cdots, q_{h}\right) \mid q_{0} \geqq 0, q_{1}+2 q_{2}+\cdots+h q_{h}=h\right\},
$$

and

(8) $C_{n m}^{(p)}\left(q_{1}, \cdots, q_{h}\right)$

where

$$
=\sum_{k=0}^{[n / p]} \sum_{S_{k}^{\prime}} \frac{(-1)^{q-t+1}}{p\left(\frac{n}{p}-k+q-t\right)}\left(\begin{array}{c}
n / p-k+q-t \\
s_{1}, \cdots, s_{h}, q_{1}-s_{1}, \cdots, q_{h}-s_{h}
\end{array}\right),
$$

$$
s_{k}^{\prime}=\left\{\left(s_{1}, \cdots, s_{h}\right) \mid 0 \leqq s_{\nu} \leqq q_{\nu}, s_{1}+2 s_{2}+\cdots+h s_{h}=k\right\}
$$

and

$$
\begin{aligned}
q & =q_{1}+q_{2}+\cdots+q_{h}, \\
t & =s_{1}+s_{2}+\cdots+s_{h} .
\end{aligned}
$$

We remark that $n / p \geqq k, q \geqq t$, hence the factor in the denominator is zero if and only if $n / p=k$, and $q=t$. But this implies $s_{1}=q_{1}$, $s_{2}=q_{2}, \cdots, s_{h}=q_{h}$, and hence $k=h$. But $n / p+m / p=h$, and since $m \geqq 1$, we cannot have $k=n / p$ and $k=h$ simultaneously. In the case when $k=n / p$, the corresponding multinomial coefficient is automatically zero as can be seen from the above calculation. Therefore in this formula, no special provision has to be made for the case when $n / p$ is an integer.

Notice that the double sum on $k$ and $S_{k}^{\prime}$ in (8) can be replaced by the single sum on $\delta=\left\{\left(s_{1}, \cdots, s_{h}\right) \mid 0 \leqq s_{\nu} \leqq q_{v}, s_{1}+2 s_{2}+\cdots+h s_{h}\right.$ $\leqq[n / p]\}$ by adding the definition $k=s_{1}+2 s_{2}+\cdots+h s_{h}$. This form would be more suitable for machine calculation.

This theorem should be compared with the result of Schur in [4]. There he obtains an explicit formula for the coefficients $C_{n k}^{(1)}$ which appears quite different from those obtained here.

A simple consequence of Theorem 4 is

TheOREM 5. $C_{N n, N m}^{(N p)}=(1 / N) C_{n, m}^{(p)}$.

PROOF. $n+m=h p$ if and only if $N n+N m=h(N p)$. Therefore, the sets $Q_{h}$ appearing in (7) are identical for these two. Since $n / p$ 
$=N n / N p$, formula (8) is identical in the two cases except that $1 / p$ is replaced by $1 / N p$ when computing $C_{N n, N m}^{(N p)}$.

From Theorem 4, we see that $C_{n m}^{(p)}$ is zero when $n+m$ is not divisible by $p$, and that the form of $C_{n m}^{(p)}$ depends only on $h$ when $n+m=h$. Thus we need consider only the $C_{n m}^{(p)}$ of the form $C_{n p-k, k}^{(p)}, k=1, \cdots$, $h p-1 ; h=1,2, \cdots$ in order to know all of the nonzero Grunsky coefficients. Calculations from the formulas of Theorem 4 suffice to give the results in the table. One example will suffice to show how these calculations may be made.

The coefficient of $a_{2} a_{3}^{2}$ in $C_{5 p-k, k}^{(p)}$ is $C_{5 p-k, k}^{(p)}(1,2,0,0,0)$. When $3<k / p<4,1<(5 p-k) / p<2$, hence

$$
C_{b p-k, k}^{(p)}(1,2,0,0,0)=\sum_{\nu=0}^{1} \sum_{S_{\nu}^{\prime}} \frac{(-1)^{3-t+1}}{p\left(\frac{n}{p}-\nu+3-t\right)}\left(\begin{array}{c}
\frac{n}{p}-\nu+3-t \\
s_{1}, s_{2}, 1-s_{1}, 2-s_{2}
\end{array}\right) .
$$

Here $n / p=5-x$. When $\nu=0, S_{\nu}^{\prime}=\{(0,0)\}$ and when $\nu=1, S_{\nu}^{\prime}$ $=\{(1,0)\}$. Thus for $3<x<4$

$$
\begin{aligned}
C_{5 p-k, k}^{(p)}(1,2,0,0,0) & =\frac{(-1)^{4}}{p(8-x)}\left(\begin{array}{c}
8-x \\
0,0,1,2
\end{array}\right)+\frac{(-1)^{3}}{p(6-x)}\left(\begin{array}{c}
6-x \\
1,0,0,2
\end{array}\right) \\
& =\frac{1}{p} \frac{(7-x)(6-x)}{2}-\frac{1}{p} \frac{(5-x)(4-x)}{2} \\
& =\frac{1}{p}(11-2 x) .
\end{aligned}
$$

Since $C_{h p-k, k}^{(p)}=C_{k, h p-k}^{(p)}$ each calculation can be checked two ways. Furthermore, the calculations are much shorter when the first index is smaller than the second and are generally simpler in the form $C_{k, h-p k}^{(p)}$. Because of this same symmetry, it suffices to make the calculations only for $k \leqq[h / 2]$ (or $k \geqq[h / 2]$ ). Since $C_{h p-k, k}^{(p)}=C_{k, h p-k}^{(p)}$ $=C_{h p-(h p-k), h p-k}^{(p)}$, the coefficients calculated above are symmetric (in $x$ ) with respect to $x=h / 2$.

Finally, we show how the inequalities of Theorem 3 can be used. It seems to be impossible to prove that $\left|a_{3}\right| \leqq 3$ by elementary use of the Grunsky inequalities, but if we put the values given below into inequality (6), assuming that $x=k / p<1$, we have $(p-k)\left|C_{p-k, k}^{(p)}\right|^{2}$ $+(2 p-k)\left|C_{2 p-k, k}^{(p)}\right|^{2} \leqq 1 / k$ or 


\section{TABLE OF $C_{n m}^{(p)}$ FOR $h \leqq 5$}

In the following, let $x=k / p$. Then

$$
\begin{aligned}
C_{p-k_{, k}}^{(p)} & =\frac{1}{p} a_{2} \quad 0<x<1 \\
C_{2 p-k, k}^{(p)} & =\frac{1}{p}\left[a_{3}-\frac{1}{2}(1+x) a_{2}^{2}\right] \quad 0<x \leqq 1,=\frac{1}{p}\left[a_{3}-\frac{1}{2}(3-x) a_{2}^{2}\right] \quad 1 \leqq x<2 \\
C_{3 p-k_{, k}}^{(p)} & =\frac{1}{p}\left[a_{4}-(1+x) a_{2} a_{3}+\frac{1}{6}(1+x)(2+x) a_{2}^{8}\right] \quad 0<x \leqq 1 \\
& =\frac{1}{p}\left[a_{4}-2 a_{2} a_{3}+\frac{1}{3}\left(1+3 x-x^{2}\right) a_{2}^{8}\right] \quad 1 \leqq x \leqq 2 \\
& =\frac{1}{p}\left[a_{4}-(4-x) a_{2} a_{3}+\frac{1}{6}(4-x)(5-x) a_{2}^{8}\right] \quad 2 \leqq x<3 \\
C_{4 p-k, k}^{(p)} & =\frac{1}{p}\left[a_{5}-(1+x) a_{2} a_{4}-\frac{1}{2}(1+x) a_{3}^{2}+\frac{1}{2}(1+x)(2+x) a_{2} a_{3}-\frac{1}{24}(1+x)(2+x)(3+x) a_{2}^{1}\right] \\
& =\frac{1}{p}\left[a_{5}-2 a_{2} a_{4}-\frac{1}{2}(1+x) a_{3}^{2}+\frac{1}{2}\left(2+5 x-x^{2}\right) a_{2}^{2} a_{3}-\frac{1}{8}(1+x)\left(2+3 x-x^{2}\right) a_{2}^{4}\right] \quad 1 \leqq x \leqq 2 \\
& =\frac{1}{p}\left[a_{5}-2 a_{2} a_{4}-\frac{1}{2}(5-x) a_{3}^{2}+\frac{1}{2}\left(6+3 x-x^{2}\right) a_{2}^{2} a_{3}-\frac{1}{8}(5-x)\left(-2+5 x-x^{2}\right) a_{2}^{4}\right] \\
& =\frac{1}{p}\left[a_{5}-(5-x) a_{2} a_{4}-\frac{1}{2}(5-x) a_{3}^{2}+\frac{1}{2}(5-x)(6-x) a_{2}^{2} a_{3}-\frac{1}{24}(5-x)(6-x)(7-x) a_{2}^{4}\right]
\end{aligned}
$$

$$
\begin{array}{rlrl}
C_{b p-h_{0}, k}^{(p)}=\frac{1}{p} & {\left[a_{6}-(1+x) a_{2} a_{5}-(1+x) a_{3} a_{4}+\frac{1}{2}(1+x)(2+x) a_{2}^{2} a_{4}+\frac{1}{2}(1+x)(2+x) a_{2} a_{3}^{2}\right.} & \\
& \left.-\frac{1}{6}(1+x)(2+x)(3+x) a_{2}^{3} a_{3}+\frac{1}{120}(1+x)(2+x)(3+x)(4+x) a_{2}^{5}\right] \quad 0<x \leqq 1
\end{array}
$$$$
=\frac{1}{p}\left[a_{6}-2 a_{2} a_{5}-(1+x) a_{3} a_{4}+\frac{1}{2}\left(2+5 x-x^{2}\right) a_{2}^{2} a_{4}+(1+2 x) a_{2} a_{3}^{2}\right.
$$$$
\left.-\frac{1}{3}(1+x)\left(3+4 x-x^{2}\right) a_{2}^{3} a_{3}+\frac{1}{30}(1+x)(2+x)\left(3+3 x-x^{2}\right) a_{2}^{5}\right] \quad 1 \leqq x \leqq 2
$$

$$
\begin{gathered}
=\frac{1}{p}\left[a_{6}-2 a_{2} a_{5}-3 a_{3} a_{4}+4 a_{2}^{2} a_{4}+\left(-1+5 x-x^{2}\right) a_{2} a_{3}^{2}-\frac{1}{2}\left(-4+15 x-3 x^{2}\right) a_{2}^{3} a_{3}\right. \\
\left.+\frac{1}{20}\left(4+25 x^{2}-10 x^{3}+x^{4}\right) a_{2}^{5}\right]
\end{gathered}
$$

$2 \leqq x \leqq 3$

$=\frac{1}{p}\left[a_{6}-2 a_{2} a_{5}-(6-x) a_{3} a_{4}+\frac{1}{2}\left(2+5 x-x^{2}\right) a_{2}^{2} a_{4}+(11-2 x) a_{2} a_{3}^{2}\right.$

$$
\left.-\frac{1}{3}(6-x)\left(-2+6 x-x^{2}\right) a_{2}^{8} a_{2}+\frac{1}{30}(6-x)(7-x)\left(-7+7 x-x^{2}\right) a_{2}^{5}\right] \quad 3 \leqq x \leqq 1
$$$$
=\frac{1}{p}\left[a_{6}-(6-x) a_{2} a_{6}-(6-x) a_{3} a_{4}+\frac{1}{2}(6-x)(7-x) a_{2}^{2} a_{4}+\frac{1}{2}(6-x)(7-x) a_{2} a_{3}^{2}\right.
$$

$$
\left.-\frac{1}{6}(6-x)(7-x)(8-x) a_{2}^{3} a_{3}+\frac{1}{120}(6-x)(7-x)(8-x)(9-x) a_{2}^{8}\right] \quad 4 \leqq x<5 .
$$




$$
\frac{p-k}{p^{2}}\left|a_{2}\right|^{2}+\frac{2 p-k}{p^{2}}\left|a_{3}-\frac{1}{2}(1+x) a_{2}^{2}\right|^{2} \leqq \frac{1}{k}
$$

which gives (since $x=k / p$ )

$$
\left|a_{3}\right| \leqq \frac{1}{2}(1+x)\left|a_{2}\right|^{2}+\left[\frac{1}{x(2-x)}-\frac{(1-x)}{(2-x)}\left|a_{2}\right|^{2}\right]^{1 / 2} .
$$

The expression on the right-hand side assumes its maximum as a function of $\left|a_{2}\right|^{2}$ when

$$
\left|a_{2}\right|^{2}=\frac{1}{x(1-x)}-\frac{(1-x)}{(2-x)(1+x)^{2}}=\mu(x) .
$$

Thus we have that

$$
\begin{aligned}
\left|a_{3}\right| & \leqq 2(1+x)+\frac{|1-2 x|}{[x(2-x)]^{1 / 2}} & \text { if } \mu(x) \geqq 4 \\
& \leqq \frac{1+2 x-x^{2}}{x(1-x)(2-x)(1+x)} & \text { if } \mu(x)<4,
\end{aligned}
$$

these inequalities being valid for any $x, 0<x<1$. In particular, if $x=0.424, \mu(x)<4$ and we have

$$
\left|a_{3}\right| \leqq 3.0437 \text {. }
$$

This appears to be about the best estimate which can be obtained for $\left|a_{3}\right|$ from the Grunsky inequalities.

The author wishes to thank the referee for calling his attention to references [4] and [5].

\section{REFERENCES}

1. Z. Charzynski and M. Schiffer, $A$ new proof of the Bieberbach conjecture of the fourth coefficient, Tech. Rep. No. 85, Stanford Univ., Stanford, Calif., 1960.

2. H. Grunsky, Koeffizientenbedingungen für schlicht abbildende meromorphe Funktionen, Math. Z. 45 (1939), 29-61.

3. M. Schiffer, Faber polynomials in the theory of univalent functions, Bull. Amer. Math. Soc. 54 (1948), 503-517.

4. I. Schur, On Faber polynomials, Amer. J. Math. 67 (1945), 33-41.

5. W. Wolibner, Sur certaines conditions nécessaires et suffisantes pour qu'une fonction analytique soit univalente, Colloq. Math. 2 (1951), 249-253.

UNIVERSITY OF MARYLAND 